\title{
Odontologia no PET-Saúde: pesquisa e integração ensino, serviço e comunidade
}

Wanda Terezinha Garbelini Frossard*, Lucimar Aparecida Britto Codato*, Maura Sassahara Higasi*, Márcia Maria Benevenuto de Oliveira*, João Paulo Menck Sangiorgio**

\author{
* Docente e tutora do Projeto PET-saúde da Universidade Estadual \\ de Londrina \\ ** Pós-graduando do Programa de Mestrado em Clínica Odontológica \\ da Universidade Estadual de Londrina
}

\section{RESUMO}

O objetivo deste trabalho é mostrar ações acadêmicas assertivas fora do âmbito da academia no curso de Odontologia da Universidade Estadual de Londrina (UEL) com a participação dos acadêmicos da área da saúde no Programa de Educação pelo Trabalho para a Saúde (Pet-saúde). Em 2010, o programa Pet-Saúde UEL aconteceu em três etapas: a territorialização da área de atuação, o estudo do referencial teórico sobre a prática do aleitamento materno e a participação dos estudantes na coleta de dados nacional sobre práticas alimentares no primeiro ano de vida. Em seguida, ocorreu a avaliação do programa e a divulgação dos trabalhos realizados em eventos científicos. Em relação ao inquérito realizado, verificou-se que: os índices de aleitamento materno exclusivo e aleitamento materno predominante estão próximos de $50 \%$; o consumo de alimentos com açúcar é elevado para a faixa etária estudada e que o uso da chupeta e da mamadeira interfere no processo da amamentação em crianças menores de seis meses de idade, favorecendo o desmame precoce $(p=0,00)$. Além disso, concluiu-se que a participação dos estudantes do curso de odontologia da UEL no Projeto Pet-saúde tem favorecido transformações nos processos de geração de conhecimentos, contribuído para a prestação de serviços à população e possibilitado maior integração entre ensino-serviço e a comunidade.

\section{DESCRITORES}

Educação Superior. Educação em Odontologia. Aleitamento Materno. $\mathbf{0}$ s desafios impostos pelas Diretrizes Curriculares Nacionais (DCN), aprovadas em 2002, e a necessidade de avançar em relação à qualificação do egresso $^{1}$ fez com que o Curso de Odontologia da Universidade Estadual de Londrina (UEL) realizasse mudanças em 2004, com a adoção de um currículo integrado, visando à formação ampliada do aluno, a interdisciplinaridade e a atenção integral.

É fato que a formação acadêmica para o curso de Odontologia deve estar atrelada a referenciais epidemiológicos, sociais, econômicos e culturais de seu meio, dirigindo sua atuação para a transformação dessa realidade em benefício da sociedade. ${ }^{2,3,4}$

Neste sentido, o curso tem buscado a diversificação de cenários de práticas por meio do fortalecimento da integração ensino-serviço e comunidade para avançar em relação à formação acadêmica em consonância com a realidade dos serviços de saúde.

Um desses cenários foi à adesão do curso de odontologia da UEL ao Programa de Educação pelo Trabalho para a Saúde (Pet-Saúde), que tem facilitado o aprendizado e a iniciação ao trabalho, por meio de estágios, que favorecem a formação profissional em consonância com as necessidades da população. $\mathrm{O}$ Pet-saúde é financiado pelos Ministérios da Saúde e Educação e, desde seu primeiro Edital, em 2009, os cursos da área da saúde da UEL têm participado ativamente das atividades do referido programa.

O Programa Pet- saúde UEL, no ano de 2010 e 2011, além de buscar a formação profissional nos locais onde efetivamente ocorrem as práticas de saúde, ${ }^{5}$ objetivou contribuir com a redução da morbimortalidade infantil por meio da ampliação da prática do aleitamento materno e da melhora da formação profissional dos cincos cursos da área da saúde da UEL 
na rede primária de atenção à saúde dos municípios de Cambé, Ibiporã e Londrina, localizados na região norte do PR.

O Objetivo deste trabalho é mostrar ações acadêmicas assertivas fora do âmbito da academia no curso de Odontologia da UEL.

\section{METODOLOGIA}

O Pet-saúde da UEL é composto por estudantes de graduação dos cursos de enfermagem, farmácia e bioquímica, fisioterapia, medicina e odontologia, regularmente matriculados nas últimas séries, totalizando 300 estudantes. O número de estudantes do curso de odontologia que participam são 120 e desses, 43 são monitores. Além deles, compõe o grupo 60 preceptores, que são profissionais pertencentes às equipes da Estratégia Saúde da Família (ESF) que orientam os alunos em suas Unidades Básicas de Saúde (UBS) e 10 tutores acadêmicos, professores da UEL, que produzem ou orientam a produção de conhecimento na área de atenção básica à saúde. O grupo Pet-saúde da UEL de Londrina, Cambé e Ibiporã é composto por 370 participantes.

Em 2010, o programa Pet-saúde da UEL aconteceu em três etapas:

- a territorialização,

- o estudo do referencial teórico sobre a prática do aleitamento materno e a participação dos alunos na coleta de dados nacional sobre práticas alimentares no primeiro ano de vida e

- a avaliação do programa e divulgação dos trabalhos realizados em eventos científicos.

\section{$1^{\text {a }}$ fase}

Inicialmente, a fim de conhecerem a área de abrangência das UBS em que atuavam, os estudantes de odontologia, juntamente com preceptores e alunos de outros cursos da área da saúde, realizaram a territorialização da região, por meio de passeios ambientais, conversas com agentes comunitários de saúde e informantes chaves. Além disso, acessaram os principais sistemas de informação sobre a Atenção Básica de seu território para maior conhecimento da prevalência e incidência dos diversos agravos na região de sua atuação.

\section{$2^{a}$ fase}

Os discentes aprofundaram-se no estudo do referencial teórico sobre as práticas do aleitamento materno, foram treinados para aplicar um questionário, já validado, sobre práticas alimentares das crianças no primeiro ano de vida, que faz parte do Projeto Amamentação e Municípios do Instituto de Saúde de São Paulo, ${ }^{6}$ nos municípios de Londrina, Cambé e Ibiporã. A participação dos alunos do curso de Odontologia na pesquisa de campo ocorreu em agosto de 2010, durante a segunda etapa da campanha de vacinação contra poliomielite, em conjunto com os estudantes dos cursos de medicina, fisioterapia, enfermagem, farmacie e bioquímica. Para o cálculo das amostras nas três localidades foi considerado a prevalência da amamentação em cada município integrante do projeto, o número de Postos de Vacinação e o número de crianças menores de um ano que foram vacinadas em cada UBS, tendo como base as planilhas de Campanhas de Vacinação do ano anterior. $\mathrm{O}$ instrumento para a coleta de dados foi composto por questões fechadas sobre o consumo nas últimas 24 horas de leite materno, outros tipos de leite e outros alimentos, incluindo água, chás e outros líquidos além de hábitos de sucção não nutritiva e características da população. Os sujeitos de pesquisa foram os acompanhantes das crianças menores de um ano de idade, no dia Nacional de Vacinação, que concordaram em participar da mesma, após a leitura do consentimento livre-esclarecido. Este estudo recebeu parecer favorável do Comitê de Ética em Pesquisa.

Além disso, os discentes levantaram nas UBS em que atuavam, crianças menores de 6 meses e fizeram o monitoramento do aleitamento materno, por meio de visitas semanais às mães com o propósito de estimulá-las para a prática do aleitamento materno.

\section{$3^{\mathbf{a}}$ fase}

No final do ano letivo de 2010, o programa foi avaliado pelos acadêmicos, preceptores e tutores através de um questionário com questões abertas e fechadas direcionado para cada modalidade de participante.

\section{RESULTADOS E DISCUSSÃO}

A participação dos estudantes do curso de Odontologia da UEL no Projeto Pet-saúde tem possibilitado transformações nos processos de geração de conhecimentos, contribuindo para a prestação de serviços à população e possibilitado maior integração entre ensino-serviço e a comunidade.

Tal integração é fundamental e indispensável para a implementação das DCN, pois para aprender saúde, como base na realidade socioeconômica, cultural e sanitária de cada território é preciso participar dos espaços onde se produz saúde. ${ }^{3,5,7,8}$ Nesse sentido, a 
Tabela 1 - Avaliação do programa Pet-Saúde pelos estudantes e preceptores participantes de Londrina, Cambe e Ibiporã/PR, em 2010.

\begin{tabular}{|c|c|c|c|c|}
\hline \multirow{2}{*}{ O Pet-Saúde possibilitou: } & \multicolumn{2}{|c|}{ Estudantes } & \multicolumn{2}{|c|}{ Preceptores } \\
\hline & CT & CNT & CT & CNT \\
\hline Aproximar-se mais à realidade dos serviços de saúde & 79,9 & 18,4 & 95,8 & 4,2 \\
\hline Conhecer melhor a realidade de vida da população & 84,6 & 12,0 & 87,5 & 1,2 \\
\hline Adquirir noções sobre os sistemas de informações epidemiológicas do município & 78,5 & 14,4 & 72,9 & 27,1 \\
\hline Conhecer os principais indicadores de saúde criança & 57,5 & 32,4 & 77,1 & 22,9 \\
\hline Conhecer as principais ações referentes à saúde da crianças desenvolvidas na rede básica de saúde & 64,1 & 27,1 & 72,9 & 27,1 \\
\hline Facilidade na relação com os alunos das outras áreas da saúde & 65,3 & 26,5 & 79,2 & 20,8 \\
\hline
\end{tabular}

CT - Concordo totalmente / CNT - Concordo, mas não totalmente.

realização do processo de territorialização, realizada na primeira fase do programa, foi fundamental para o conhecimento da realidade e das necessidades de cada região.

Somado a isso, o fato do processo ensino-aprendizagem ocorrer nos espaços onde as práticas de saúde acontecem, favorece a formação de profissionais mais sensíveis, com maior comprometimento para o enfrentamento das reais necessidades de saúde da população, pois o aluno tem a oportunidade de vivenciar e participar do dia-dia dos serviços. ${ }^{2,3,5,7}$

Além disso, o aprendizado e o trabalho conjunto entre estudantes, preceptores e tutores de outras áreas da saúde, têm auxiliado uma maior integração entre os acadêmicos dessas áreas, como mostra os resultados da avaliação do programa realizado pelos estudantes e preceptores (Tabela 1)

Na segunda fase do programa, os estudantes participaram da pesquisa de campo nos Postos de vacinação durante o Dia Nacional da Campanha de Vacinação e, do total da amostra estimada, realizaram $88,91 \%, 92,2 \%$ e $93,06 \%$ entrevistas com mães ou acompanhantes de crianças menores de um ano de idade em Londrina, Cambé e Ibiporã, respectivamente, totalizando nos três municípios 2723 questionários respondidos.

Os resultados mostraram em crianças menores de seis meses de idade que o aleitamento materno exclusivo (AME) foi de 40,56\%, 41,12\% e 42,91\% em Londrina, Cambé e Ibiporã, respectivamente. Quando somado ao aleitamento materno predominante (AMP) este índice foi de 52,81\%, 51,98\% e 52,70\% em Londrina, Cambé e Ibiporã, respectivamente.

Os dados obtidos de AME e AMP, quando comparados ao trabalho de Vannuchi et al. ${ }^{9}$ (2005), mostram que, apesar do aumento significativo de $15 \%$ ao longo da última década no município de Londrina-PR, estes dados ainda estão aquém do preconizado pela Organização Mundial da Saúde para o milênio. ${ }^{9}$

O uso da chupeta e da mamadeira, além de contribuir para as más oclusões na dentadura decídua, ${ }^{10,11,12,13,14}$ mostrou que interfere no processo da amamentação em crianças menores de seis meses de idade, favorecendo o desmame precoce nas três localidades $(p=0,00)$. Esses achados estão de acordo com os estudos de Cotrim et al..$^{12}$ (2002), em São Paulo e Soares et al. ${ }^{13}$ (2003) no Rio de Janeiro.

O consumo de alimentos adoçados e a ingestão de refrigerantes ocorreu em 33,89\%, 30,74\% e $33,45 \%$ e em $8,11 \%, 7,26 \%$ e $10,96 \%$ em Londrina, Cambé e Ibiporã, respectivamente. Esses dados são preocupantes, uma vez que a dieta rica em sacarose, um dos responsáveis pela cárie dentária, ${ }^{14,15}$ mostra-se elevado para a faixa etária estudada.

\section{CONCLUSÃo}

A participação de alunos do Curso de Odontologia da UEL no programa Pet-saúde mostrou-se efetiva para o processo ensino-aprendizagem, favoreceu o reconhecimento da realidade da população, possibilitou o trabalho multiprofissional e permitiu que vivenciassem a pesquisa.

O monitoramento das mães em aleitamento materno, estimulando a prática, foi a estratégia encontrada pelos acadêmicos do programa para contribuir com a redução da mortalidade infantil.

\section{ABSTRACT}

Dentistry in the "PET-Saúde" Project: research and integration of teaching, service and community

The aim of this study was to show the assertive academic actions performed outside the academic scope of the dentistry course at the State University of Londrina (UEL), with the participation of health 
field students enrolled in the Tutorial Education Program (PET) promoted by the Health and Education Ministry. In 2010, the "PET-Saúde UEL" program was conducted in three stages: marking off the work area, theoretical reference study on the breastfeeding practice, and participation of students in gathering national data on eating habits in the first year of life. Next, the program was evaluated and the studies presented in scientific events were disseminated. Regarding the survey, it was found that: the rates of exclusive and predominant breastfeeding are close to $50 \%$, that the consumption of sugar-based foods is high for the age group studied, and that the use of pacifiers and bottles interferes with the process of breastfeeding in children under six months of age and favors early weaning ( $p=0.00$ ). Furthermore, it was concluded that the participation of dentistry students in the "PET-Saúde UEL" project has promoted changes in the processes of broadening knowledge, contributing to public services and enabling greater integration between teaching, health service and community.

\section{DESCRIPTORS}

Higher Education. Dental Education. Breast feeding.

\section{REFERÊNCIAS BIBLIOGRÁFICAS}

1. Brasil. Ministério da Educação-CNE. Conselho Nacional de Educação. Resolução CNE-CES 3 de 19/02/2002. Institui as Diretrizes Curriculares Nacionais do Curso de Graduação em Odontologia. Diário Oficial da União, Brasília, 04 de março de 2002.

2. Morita MC, Kriger L. A relação ensino e serviços de saúde. In Perri de Carvalho AC; Kríger, L. Educação Odontológica. São Paulo: Artes Médicas, 2006.

3. Moyses SJ. Intersetorialidade e Multidisciplinaridade como desafios para a Odontologia. In: Pereira AC. Tratado de Saúde Coletiva. Nova Odessa: Napoleão, 2009.

4. Junqueira SR, Frias AC, Araújo ME, Zara EB. Visitas supervisionadas aos serviços de saúde como estratégia pedagógica: percepções de alunos de Odontologia. Revista Espaço para a Saúde 2009; 11(1): 38-47.

5. Brasil. Ministério da Saúde. Programa Nacional de Educação pelo Trabalho para a Saúde-PET-SAÚDE. Acesso em 10/09/10 Disponível em: www.saude.gov.br/porta/SGTES.

6. Venâncio SI, Escuder MML, Kitoko P, Rea MF, Monteiro CA. Freqüência e determinantes do aleitamento materno em municípios do Estado de São Paulo. Rev Saúde Pública 2002; 36:313-8.

7. Brasil. Ministério da Saúde. Ministério da Educação. Programa Nacional de Reorientação da Formação Profissional em Saúde - Pró-saúde. Objetivos, Implementação e Desenvolvimento Potencial. Brasília: Ministério da Saúde, 2007.

8. Morita MC, Codato LAB, Higasi MS, Kasai MLH. Visita Domiciliar: espaço de aprendizagem na graduação em Odontologia. Revista de Odontologia da UNESP 2010; 39(2): 75-79.

9. Vannuchi MTO, Thomson Z, Escuder MML, Tacla MTGM, Vezozzo KMK, Castro LMCP, et al. . Perfil do aleitamento materno em menores de um ano no Município de Londrina, Revista Brasileira de Saúde Materno Infantil. 2005; 5(2): 155-162.

10. Viggiano D, Fasano D, Monaco G, Strohmenger L. Breast feeding, bottle feeding and non-nutritive sucking: effects on occlusion in deciduous dentition. Arch Dis Child. 2004 December; 89(12): 1121-1123.

11. Carrascoza KC, Possobon RF, Tomita LM, Moraes ABA. Consequências do uso da mamadeira para o desenvolvimento orofacial em crianças inicialmente amamentadas ao peito. J. Pediatr. 2006; 82(5): 395-97.

12. Cotrim LC, Venancio SY, Escuder MML. Uso de chupeta e amamentação em crianças menores de quatro meses no estado de São Paulo. Rev Bras Saúde Infant 2002; 2:253- 61.

13. Soares MEM, Giugliani ERJ, Braun ML, Salgado ACN, Oliveira AP, Aguiar PR. Uso de chupeta e sua relação com o desmame precoce em população de crianças nascidas em Hospital Amigo da Criança. J Pediatr. 2003; 79(4): 309-316.

14. Ismail AI, Sohn W. The impact of universal access to dental care on disparities in caries experience in children. J Am Dent Assoc. 2001; 132(3):295-303.

15. Warren JJ, Weber-Gasparoni K, Marshall TA, Drake DR, Dehkordi-Vakil F, Kolker JL, Dawson DV. Factors associated with dental caries experience in 1-year-old children.J Public Health Dent. 2008; 68(2):70-5. 